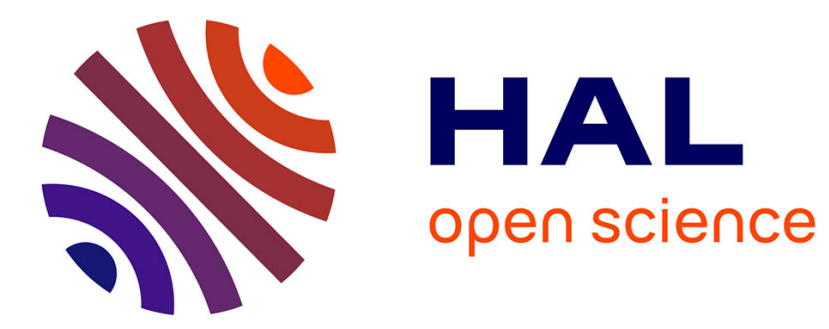

\title{
Virtual Forces based UAV Fleet Mobility Models for Air Pollution Monitoring
}

Amjed Belkhiri, Walid Bechkit, Hervé Rivano

\section{To cite this version:}

Amjed Belkhiri, Walid Bechkit, Hervé Rivano. Virtual Forces based UAV Fleet Mobility Models for Air Pollution Monitoring. LCN 2018 - 43rd Annual IEEE Conference on Local Computer Networks, IEEE, Oct 2018, Chicago, Illinois, United States. pp.1-4. hal-01956717

\section{HAL Id: hal-01956717 https://hal.inria.fr/hal-01956717}

Submitted on 16 Dec 2018

HAL is a multi-disciplinary open access archive for the deposit and dissemination of scientific research documents, whether they are published or not. The documents may come from teaching and research institutions in France or abroad, or from public or private research centers.
L'archive ouverte pluridisciplinaire HAL, est destinée au dépôt et à la diffusion de documents scientifiques de niveau recherche, publiés ou non, émanant des établissements d'enseignement et de recherche français ou étrangers, des laboratoires publics ou privés. 


\title{
Virtual Forces based UAV Fleet Mobility Models for Air Pollution Monitoring
}

\author{
Amjed Belkhiri*, Walid Bechkit* and Hervé Rivano* \\ *Univ Lyon, Inria, INSA Lyon, CITI, F-69621 Villeurbanne, France
}

\begin{abstract}
One of the main issues in UAVs networks design is how nodes are relocated in order to meet the desired performance objectives. In this work, we propose two UAVs fleet mobility models based on the Virtual Forces Algorithm (VFA). The application context we are interested in is the air pollution surveillance over wide areas. The first model is a centralized variant where all computations are performed in a central ground basestation. While the second model is a distributed version where each node takes its own decision in collaboration with its neighbors. We evaluate our models performances and we compare them with state of the art solutions using a real data set of air pollution concentrations and according to three main metrics: the maximal estimation error, execution time and communication cost.
\end{abstract}

Keywords-UAVs Network, Mobility Models, Virtual Forces Algorithm, Air quality monitoring.

\section{INTRODUCTION}

Air quality is a major concern in nowadays cities. The World Health Organization (WHO) estimates that over $92 \%$ of world's population lives in places where air pollution exceeds guidelines limits and that every year more than 7 millions deaths are associated with air pollution. [1].

Current technological advances in the field of Unmanned Air Vehicles (UAV) have enabled the Unmanned Aerial Systems (UAS) as a viable deployment platform for efficient air-born sensor networks for air pollution monitoring. Using UAV swarms offers higher performances in terms of mission duration, mission area, and ensuring a high level of cooperation and collaboration comparing to single UAV systems. Despite the challenges faced by UAS (autonomous control, mission planning, coordination...), these systems are currently used in many Search and Rescue (SAR) applications and in the study of natural phenomena [2].

In this work, we study the use of Unmanned Aerial Vehicles swarms in air pollution surveillance. We present two UAV swarm mobility models based on Virtual Forces Algorithm (VFA) that take in consideration both the physical phenomenon and the network connectivity constraints to ensure a timely and high spatial resolution surveillance. We also detail our models simulation performance analysis and compare them to some state of the art models.

\section{RELATED WORK}

In this work, we are interested in autonomous control of airborn WSN for air pollution monitoring using UAV swarms as deployment platforms. Therefore, we present in this section two categories of state of art works. First, we highlight prior works that tackled WSN deployment strategies for air quality surveillance. Then, we introduce works relative to UAV swarms autonomous control models from both generic and application oriented points of view.

\section{A. WSN deployment strategies for air quality monitoring}

WSN deployment modeling is a very active research area. In air pollution surveillance context, we distinguish two main categories of WSN deployment strategies. The first class contains static WSN deployment models, where sensors' positions are fixed in a manner to ensure accurate and high resolution air quality mapping. Boubrima et al. [3] propose an optimal integer linear programming model that computes sensors deployments under both air pollution coverage and network connectivity constraints. They applied atmospheric dispersion and estimation models to assess pollution concentrations in wide areas with a bounded estimation error.

In the second class, we find works that exploit different mobile agents as deployment platforms for pollution monitoring WSN. The authors in [4] used a predefined mobility model, where sensors are anchored to public buses. Combining the collected data and estimation models, they obtained a high resolution dynamic air pollution mapping of the city of Lausanne (Switzerland). The integration of mobility models in air pollution surveillance WSN allowed to enhance the spatiotemporal resolution and the estimation accuracy. However, the reaction to the dynamic nature and the unpredictable changes of studied phenomena and its environment was not studied.

\section{B. UAV swarms autonomous control}

Most of current approaches of UAV control systems focus on the use of single UAV. The main reason of such limitation is that formation, coordination and autonomous control in multiple UAV systems introduce additional challenges (coordination, collision avoidance...). Recently, many UAV fleet coordination and mobility control paradigms have been proposed. In this work, we are more interested in online distributed control models where UAVs are autonomous entities equipped with wireless communication capabilities and they self organize and coordinate to complete their mission. Messous et al. [5] proposed a fuzzy logic based UAVs swam mobility model for area exploration. They considered network connectivity and UAV energy as decision criterion. Based on these criterion and the information received from its neighbors, each UAV determines its next destination. UAVs swarms have been used in wide range of civil applications [6]. However, most solutions tackle the area covergae problem without considering the physical phenomena characteristics.

In contrast to the previous presented works, we are interested in UAVs swarms online decentralized mobility control models for time continuous air pollution monitoring. We aim to provide, both wide phenomena coverage and unusual event (explosions, gas leakage...) source detection and providing at least a 1-connected network according to UAVs density and the deployment environment. 


\section{III.PRELIMINARIES}

In this section, we introduce some preliminaries definitions and notions that are important to understand the application context of our contribution and the problem we are interested in resolving.

\section{A. Reference and simulation maps}

We consider the deployment region as a $3 \mathrm{D}$ virtual grid $\mathcal{P}$ of $l \times w \times h$ cubic cells. Each cell $p$, characterized by its real air pollution concentration $\mathcal{G}_{p}$ and its coordinates $\left(x_{p}, y_{p}, z_{p}\right)$, represents a potential deployment position for a UAV $u$. The set of real atmospheric pollution concentrations $\mathcal{G}_{p}$ at grid points $p$ forms the $3 \mathrm{D}$ air pollution concentrations reference map, $\mathcal{R}_{\text {map }}=\left\{\mathcal{G}_{p}, p \in \mathcal{P}\right\}$. The construction of the air pollution reference map requires punctual and time continuous measuring at each point $p$ of the deployment region, which is not feasible. Therefore, we rely on atmospheric dispersion simulations in order to estimate the concentration of pollutants with a high spatial granularity. These values form the simulation map $\mathcal{S}_{\text {map }}=\left\{\mathcal{S}_{p}, p \in \mathcal{P}\right\}$ that approximates the ground truth map $\mathcal{R}_{\text {map }}$. For each point $p$, the estimated value $\mathcal{S}_{p}$, presents a maximal simulation error that we call $S_{e_{p}}$. Hence $S_{p}$ and $S_{e_{p}}$ verify the relation: $\mathcal{S}_{p}-\mathcal{S}_{e_{p}} \leq \mathcal{G}_{p} \leq \mathcal{S}_{p}+\mathcal{S}_{e_{p}}$.

\section{B. Estimation and error maps}

In order to estimate the concentration where no UAV is deployed, we use a deterministic spatial interpolation method called IDW (Inverse Distance Weighting). This method formulates the estimated concentration $\mathcal{Z}_{p}$ at a given position $p$ where no UAV is present as a weighted combination of reference concentrations $\mathcal{S}_{q}$ measured by nodes deployed at points $q$. Note that the IDW estimation model and the distance correlation coefficient formulation promote the effect of nearest points.

Based on the estimation concentrations $\mathcal{Z}_{p}$ at all points $p \in \mathcal{P}$, we generate the air pollution estimated map $\mathcal{Z}_{\text {map }}=\left\{\mathcal{Z}_{p}, p \in\right.$ $\mathcal{P}\}$. To evaluate the estimation precision at each point $p$, This error $\mathcal{E}_{p}$ is defined as the absolute difference between reference value $\mathcal{G}_{p}$ and estimated value $\mathcal{Z}_{p}$ (i.e. $\mathcal{E}_{p}=\left|\mathcal{Z}_{p}-\mathcal{G}_{p}\right|$ ). Using estimation errors at all deployment region points, we construct a new map called error map $\mathcal{E}_{\text {map }}=\left\{\mathcal{E}_{p}, p \in \mathcal{P}\right\}$.

\section{Problem formulation}

Given an initial deployment $X^{i}$ of a set of UAVs, we aim is to find the new UAVs distribution in order to respond to the variations in reference concentration values, resulting of the dynamic nature of the air pollution phenomena. In order to find the new nodes distribution, that we call $X^{f}$, we propose new redeployment models to relocate UAVs taking into account the network connectivity and execution time.

\section{IV.OUR PROPOSAL}

In this section, we detail our UAV swarm control models for air pollution monitoring, which are two adapted of the traditional Virtual Forces Algorithm.

\section{A. Centralized Error Based Virtual Forces Algorithm}

This algorithm is based on a centralized decision making mechanism, where all computations and decisions are made by a ground decision-making entity (a sink node). We assume that the sink communication range is wide enough to cover the whole deployment area.

1) Virtual forces model: The position of each UAV $u$ is represented by its $3 \mathrm{D}$ coordinates $P_{u}=\left(x_{u}, y_{u}, z_{u}\right)$. We denote $d\left(p, P_{u}\right)$ the euclidean distance between the UAV position and the point $p$ in the deployment region. In contrast with the traditional VFA model, we consider the points $p$ with estimation errors $e_{p}>0$ as force sources instead of other UAVs. Hence, each UAV $u$ is subject to attractive forces $\vec{F}_{p u}$ applied by the set of points $S(u)=\left\{p \in \mathcal{P}, d\left(p, P_{u}\right) \leq\right.$ $\left.d_{t h} \& e_{p}>0\right\}$. Where $d_{t h}$ is predetermined virtual forces impact threshold distance. The total force applied on the UAV $u$ can be expressed as :

$$
\overrightarrow{\mathcal{F}}_{u}=\sum_{p \in \mathcal{S}(u)} \overrightarrow{\mathcal{F}}_{p u}
$$

In our model, we propose to use the normalized estimation error $e_{p} / \sum_{p \in \mathcal{S}(u)} e_{p}$ to express the point $p$ error importance in the attractive force applied on $u$. On the other side and in order to minimize the movement distance and consequently the redeployment energy, we promote the impact of nearest points $p$ by considering the proportion $d_{t h} / d\left(p, P_{u}\right)$.

Hence, we give the attractive force applied by a point $p$ on a UAV $u$ as:

$$
\begin{aligned}
\overrightarrow{\mathcal{F}}_{p u}=\left[\left(\frac{d_{t h}}{x_{p}-x_{u}}\right) \vec{u}_{x}+\left(\frac{d_{t h}}{y_{p}-y_{u}}\right) \vec{u}_{y}\right. & \left.+\left(\frac{d_{t h}}{z_{p}-z_{u}}\right) \vec{u}_{z}\right] \\
& \cdot\left(\frac{e_{p}}{\sum_{p \in \mathcal{S}(u)} e_{p}}\right)
\end{aligned}
$$

2) Movement equation: Each UAV $u$ moves following its total force's $\vec{F}_{u}$ direction until it reaches its destination where the total force becomes null. We describe sensor node movement equation as:

$$
\overrightarrow{P_{u}}\left(t_{f}=t_{i}+\Delta t\right)=\frac{\overrightarrow{\mathcal{F}}_{u}}{\left\|\mathcal{F}_{u}\right\|} \cdot \Delta t \cdot \bar{v}+\vec{P}_{u}\left(t_{i}\right)
$$

3) The Centralized Error Based Virtual Forces Algorithm: The following steps resume the core algorithm that allows to start at $t_{i}$ with an initial deployment of UAVs characterized by a maximal estimation error $e_{i}$ and gives as a result the final deployment at instant $t_{f}$ characterized with $e_{f}$ such as $e_{f}<e_{i}$. For each deployed UAV $u$ the sink node defines all virtual force sources $p$ within a $d_{t h}$ radius. The next position of $u$ is obtained using equation (3) after assessing its total attractive force $\overrightarrow{\mathcal{F}}_{u}$ according to (1) and (2). Based on its global view of the network, the sink simulates the movement of the UAVs towards their respective new positions and then evaluates the new maximal estimation error $e_{f}$. If the UAV's destination is free and $e_{i}>e_{f}$ then the movement is validated, else this movement degrade the estimation quality and it's ignored. This constraint allows to ensure convergence, 
avoid collisions during movement and limit oscillation effect caused by conflicting virtual forces. The algorithm is executed until no node can be redeployed. At the end of execution, UAVs are redeployed according to their total movement vectors constructed of valid virtual movements. The core algorithm is executed repeatedly, considering at each iteration the changes in the simulation, estimation and error maps $\left(\mathcal{S}_{\text {map }}, \mathcal{Z}_{\text {map }}\right.$ and $\left.\mathcal{E}_{\text {map }}\right)$.

\section{B. Distributed Error Based Virtual Forces Algorithm}

In this distributed variant, we assume that all the UAVs are homogeneous and they all have the same communication range denoted $d_{c}$.

At the beginning of each iteration, each UAV $u$ broadcasts its state vector to all of its neighboring UAVs. The state vector contains information about the UAV current state such as the 3D coordinates $\left(x_{u}, y_{u}, z_{y}\right)$ and the in situ measure of air pollution and the energy remaining level. In the other hand, it also receives the state vectors of all the nodes within its communication range and also the current local simulation map from the ground base station. The local simulation map is a part of the global map that contains only the air pollution concentrations in the neighborhood of the considered UAV.

Once these information are acquired, the UAV generates both the estimation and the error maps based on the measured concentrations. Then, it computes its new destination using the previous equations and considering as force sources only the points $p$ with estimation errors within its communication range $d_{c}$ (i.e. $S(u)=\left\{p \in \mathcal{P}, d\left(p, P_{u}\right)<d_{c} \& e_{p}>0\right\}$ ). Afterwards, the UAV broadcasts the computed destination and it waits until receiving all the other neighbors' destinations. Before changing its position, each UAV verifies that the movement don't degrade the estimation quality and that the UAV stay within the communication range of at one of its current neighbors. If two sensors have the same destination, a selection process is initiated. This process chooses the node to redeploy towards the conflict destination based on the shortest distance as a redeployment criterion. The selection process allows to avoid collision and to enhance the network lifetime by reducing the redeployment energy. Finally, all UAVs are relocated and they re-initiate the the neighborhood discovery and the redeployment process all over again.

\section{Simulation Results And Analysis}

The performance evaluation includes the estimation accuracy represented by the maximal estimation error after (following) a redeployment, the execution time and the communication cost. The presented results are obtained through extensive simulations on a real air pollution concentrations data set.

\section{A. Simulation assumptions and setup}

We perform our simulations using nitrogen dioxide $\left(\mathrm{NO}_{2}\right)$ concentrations maps of La Part-Dieu district, of Lyon City in France. The data set is provided by Air-Rhone-Alpes an observatory for air pollution monitoring using an atmospheric dispersion simulator called SIRANE [7]. For simplicity reasons, we consider a free of obstacles 2D deployment region.

\section{B. Performance analysis}

In order to evaluate the quality of our mobility models in regards to other existing models, different performance criteria have been proposed. Four metrics are used in this work : maximal estimation error, execution time and the communication cost.

1) Maximal estimation error: The maximal estimation error represents the accuracy of the air pollution estimation map following a redeployment. . Figure 1 illustrates the average maximal estimation error given by the initial deployment and the averages resulting of both the centralized and distributed $\left(d_{c}=1450 \mathrm{~m}\right)$ virtual forces models.

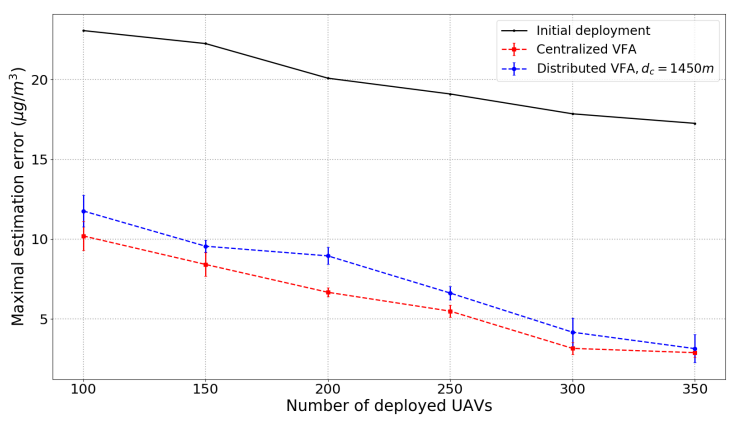

Fig. 1: Average maximal estimation error.

We observe that both the centralized and the distributed versions allow to significantly reduce the maximal estimation error. The centralized version offers better estimation enhancement since it is based on a global centralized overview of the deployment region. This difference is due to the distinction between the redeployment criteria in both versions. In the centralized VFA, the ground station privileges the global vision of the network. Therefore, the redeployed UAV is the one that minimizes better the maximal estimation error. In the distributed version, each UAV aims to reduce its redeployment energy consumption. Moreover, we notice from Figure. 1 that the two versions converge towards the same solution which explains the small differences in dense deployments.

In order to study the impact of the communication range $d_{c}$, we illustrate in the figure 2 the maximal estimation error according to different values of $d_{c}$ from $150 \mathrm{~m}$ up to $1350 \mathrm{~m}$ with an increase of $100 \mathrm{~m}$ for all different configurations (100, 150, 200, 250, 300 and 350 UAVs).

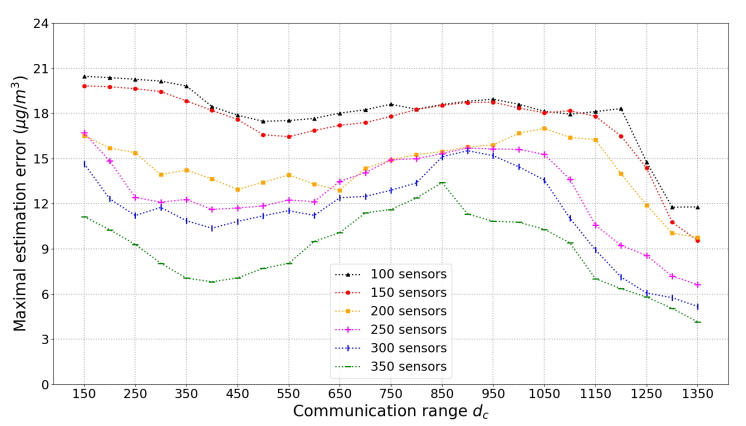

Fig. 2: Communication range impact (distributed VFA).

We notice that the simulation results have the same trend for all different configurations. At the beginning, the estimation error 
decreases with the increase of the communication range. For small values of $d_{c}$, the UAV neighborhood is restricted and as a consequence its redeployment impact is limited in its vicinity. The increase of $d_{c}$ allows the UAVs to explore wider areas and have more important impact on the estimation error. However, this increase starts to gradually have a negative impact. In wider neighborhood areas, the virtual forces sources number is more significant and their dispersion is sparser which results in creating conflicting virtual forces that limit the UAV mobility and as a result the estimation error enhancement. For bigger values of $d_{c}$, above $950 \mathrm{~m}$, more UAVs are in collaboration and they cover larger regions at once. Hence, we notice the decrease in the estimation error to lower values.

2) Execution time: We analyze in this simulation the models' execution time according to the number of deployed sensors as depicted in Fig. 3. In this work, the execution time is represented considering only the effective computational time needed to find the final destination, both the communication and movement times are ignored.

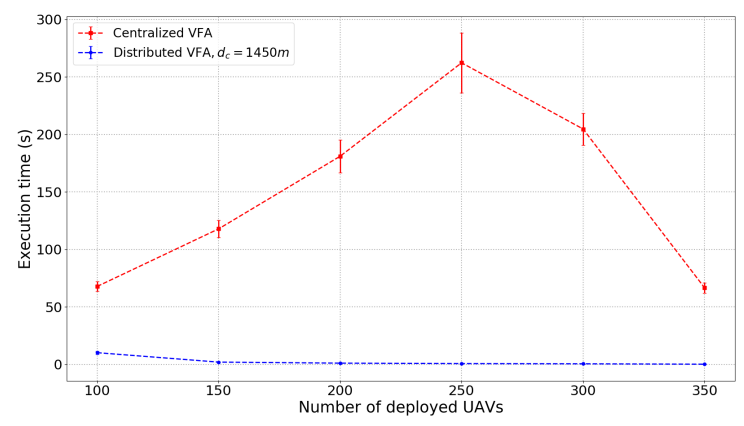

Fig. 3: Execution time centralized vs. distributed.

In sparse deployments, the number of force sources is considerably important. However, they are distant from the deployed UAVs and their forces impact is minimal. Therefore, the nodes movements are limited and the final equilibrium state of the algorithm is quickly reached. Increasing the number of deployed nodes reduces both the presence of force sources and their distances from the nodes. Hence, the sources impact on the neighboring UAVs is more important which causes more movements and a longer execution time. In denser deployments, the algorithm convergence is faster since the redeployment space is more restricted.

In the distributed version, the execution time decreases with the increase of the number of deployed UAVs. With more nodes in the deployment region, the number of force sources is reduced. Therefore, the computational time spent by each UAV to assess the virtual forces and the final destination is less significant. The distributed variant is at least 6 times faster in comparison with the centralized model.

3) Communication cost: The figure 4 shows the average number of transmissions per UAV node according to the communication range $d_{c}$. We first notice that all the configurations have the same trend. For small communication ranges, the number of each UAV neighbors is limited and its movements are restricted. Therefore, the transmissions number during the two phases of neighborhood discovery and redeployment is low. Enlarging the $d_{c}$ distance increases the number of transmissions in both phases and consequently the global average.
However, for $d_{c}$ between $650 \mathrm{~m}$ and $950 \mathrm{~m}$ the number of transmissions decreases due to the drop-off in nodes mobility caused by the conflicting virtual forces as explained before. Once, the nodes have a better overview on the deployment area $\left(d_{c}>950 \mathrm{~m}\right)$, the transmission average increases again since both the UAVs neighborhood and their movements are less restricted.

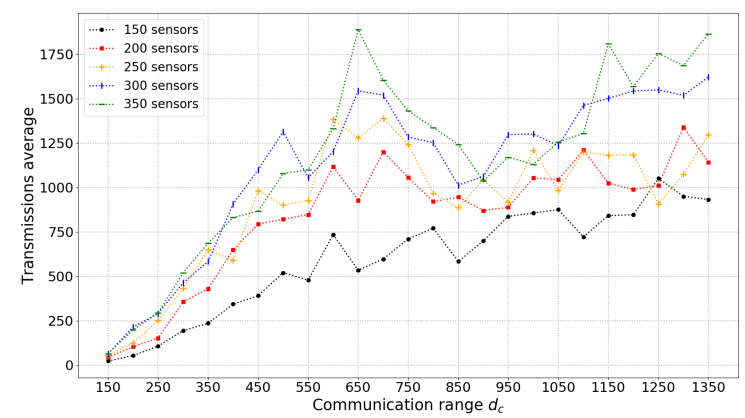

Fig. 4: Transmissions average vs. communication range $d_{c}$.

\section{CONCLUSION}

In this work, we proposed two virtual forces based models for UAVs autonomous fleet control suitable for area coverage applications. Both proposed models (centralized and distributed) aim to achieve an accurate global estimation of air pollutant concentrations on the deployment region. In the centralized version, all computations and control operations are handled by the base stations. In the distributed variant, the computation tasks are delegated to the nodes. Performance analysis showed that both models reduce the maximal estimation error to at least $40 \%$ of its initial value. While the first model offers a better maximal estimation error the distributed model is less time consuming. However, the latter has a major drawback in terms of communications.

\section{REFERENCES}

[1] World Health Organisation. WHO: Ambient (outdoor) air quality and health, publié: 2016-09. Accès: 2017-05.

[2] Brian A White, Antonios Tsourdos, Immanuel Ashokaraj, S Subchan, and Rafal Zbikowski. Contaminant cloud boundary monitoring using network of uav sensors. IEEE Sensors Journal, 8(10):1681-1692, 2008.

[3] Ahmed Boubrima, Walid Bechkit, and Hervé Rivano. Optimal wsn deployment models for air pollution monitoring. IEEE Transactions on Wireless Communications, 16(5):2723-2735, 2017.

[4] Ali Marjovi, Adrian Arfire, and Alcherio Martinoli. High resolution air pollution maps in urban environments using mobile sensor networks. In Distributed Computing in Sensor Systems (DCOSS), 2015 International Conference on, pages 11-20. IEEE, 2015.

[5] Mohamed-Ayoub Messous, Sidi-Mohammed Senouci, and Hichem Sedjelmaci. Network connectivity and area coverage for uav fleet mobility model with energy constraint. In Wireless Communications and Networking Conference (WCNC), 2016 IEEE, pages 1-6. IEEE, 2016.

[6] Samira Hayat, Evşen Yanmaz, and Raheeb Muzaffar. Survey on unmanned aerial vehicle networks for civil applications: A communications viewpoint. IEEE Communications Surveys \& Tutorials, 18(4):26242661, 2016.

[7] Lionel Soulhac, Pietro Salizzoni, Patrick Mejean, D Didier, and I Rios. The model sirane for atmospheric urban pollutant dispersion; part ii, validation of the model on a real case study. Atmospheric environment, 49:320-337, 2012. 